\section{MELANOTIC NEUROECTODERMAL TUMOUR OF INFANCY: UNUSUAL HISTOLOGICAL FEATURES IN A RARE TUMOUR}

\author{
Julia Low, Helen Doyle, Erica Jacobson, Martin Weber \\ Anatomical Pathology, Prince of Wales Hospital, Randwick, \\ NSW, Australia
}

Description: Melanotic neuroectodermal tumour of infancy (MNTI) is a rare, locally aggressive but usually benign tumour typically arising in the bones of the head and neck in infants and young children. Histologically, MNTI is a biphasic tumour of neural crest origin, composed of neuroblastic and pigmented epithelial components.

Findings: We present a case arising from the skull of an 18month-old boy requiring extensive neurosurgical resection. Histological examination confirmed MNTI but with unusual features, viz: (a) the 'small-round-blue-cell' neuroblastic component, usually bland with rare mitoses, showed morphological features suggestive of malignancy, including abundant mitoses (up to 67/10 hpf) and karyorrhexis, foci of necrosis, and a high Ki67 proliferative index ( $40 \%)$; and (b) the neuroblastic component, in addition to showing typical 'small-round-bluecell' morphology, included large areas of neuropil containing neuroblastic cells showing varying degrees of ganglionic differentiation.

Discussion: Less than 500 MNTIs are reported in the published literature; only $7 \%$ are malignant, the latter based on the presence of metastatic disease. ${ }^{1}$ There are currently no defined histological criteria for malignant MNTI, ${ }^{2}$ and there was no evidence of metastatic disease in our patient. Ganglionic differentiation is described in $\mathrm{MNTI}^{3,4}$ but, to our knowledge, this usually constitutes a minor component.

\section{References}

1. Fowler DJ, Chisholm J, Roebuck D, et al. Melanotic neuroectodermal tumor of infancy: clinical, radiological, and pathological features. Fetal Pediatr Pathol 2006; 25: 59-72.

2. Kapadia SB, Frisman DM, Hitchcock CL, et al. Melanotic neuroectodermal tumor of infancy: clinicopathological, immunohistochemical, and flow cytometric study. Am J Pathol 1993; 17: 566-73.

3. Reddy ER, Kumar MS, Aduri R, et al. Melanotic neuroectodermal tumor of infancy: A rare case report. Contemp Clin Dent 2013; 4: 559-62.

4. Shah RV, Jambhekar NA, Rana DN, et al. Melanotic neuroectodermal tumor of infancy: report of a case with ganglionic differentiation. $J$ Surg Oncol 1994; 55: 65-8.

\section{SYNCHRONOUS SESSILE SERRATED ADENOMA AND GOBLET CELL CARCINOID IN THE APPENDIX: A CASE REPORT AND LITERATURE REVIEW}

Zheqin R. Lu, Phillip Jayasurya

Dorevitch Pathology, Traralgon, Vic, Australia

Introduction: Appendiceal neoplasms are rare, with the majority being adenomas and neuroendocrine tumours. Sessile serrated adenoma (SSA) is associated with the serrated pathway of neoplasia. Less than 50 cases of SSA in the appendix have been reported. Goblet cell carcinoid (GCC) is almost exclusively found in the appendix and has a worse prognosis than other carcinoid variants. We present an interesting case of synchronous SSA and GCC within the appendix.

Case report: A 49-year-old male presented with acute right iliac fossa pain. The appendicectomy specimen measured $55 \mathrm{~mm}$ in length and $12 \mathrm{~mm}$ in diameter. Histopathology showed irregular serrated glands, cryptal branching and dilated ' $L$ ' shaped crypt bases in the mucosa. There was a second infiltrating tumour within the muscularis propia composed of discrete clusters of goblet like cells. Immunohistochemically, the SSA and GCC showed positive staining for AE1/3, CK20 and polyclonal CEA. The goblet cell tumour was also positive for synaptophysin and CD56.

Discussion: This case of two rare synchronous appendiceal neoplasms highlights the importance of adequate sampling. Although SSAs are rarely described in the appendix, it should be recognised due to a higher risk of malignant transformation. It is further important to differentiate appendiceal goblet cell carcinoid from the more aggressive mucinous adenocarcinoma.

\section{MALIGNANT PROLIFERATING TRICHILEMMAL TUMOUR: A CASE REPORT AND LITERATURE REVIEW}

\author{
Zheqin R. Lu, Gregory Imhagwe \\ Dorevitch Pathology, Traralgon, Vic, Australia
}

Introduction: Malignant proliferating trichilemmal tumour (MPTT) is a rare neoplasm with less than 50 cases documented in the literature. It is more common on the scalp and in females. We report a case of MPTT and review the histopathology, differential diagnoses and management.

Case report: An 84-year-old male presented with a tan subcutaneous scalp nodule and underwent an elliptical skin excision $(30 \times 22 \times 15 \mathrm{~mm})$. Histopathology showed a well circumscribed dermal mass with a multi-loculated cystic appearance. It was lined by squamous epithelium with an absent granular layer showing evidence of abrupt keratinisation. The keratinocytes showed marked cytologic atypia. Numerous mitoses including atypical mitotic figures were seen. The lesion extended to the margins. The morphological features were consistent with a MPTT.

Discussion: It is important to recognise this rare entity as MPTT tends to recur and metastasise more frequently than the histological mimics of squamous cell carcinoma (SCC) and trichilemmal carcinoma (TLC). Features favouring MPTT over SCC include presence of trichilemmal type keratinisation and the lack of a premalignant epidermal lesion. TLC is distinguished by a lobular proliferation of clear cells and continuity with the epidermis. Wide excision of MPTT with an adequate clearance margin and close follow up are the mainstays of treatment.

\section{References}

1. Ye J, Nappi O, Swanson PE, et al. Proliferating pilar tumors: A clinicopathologic study of 76 cases with a proposal for definition of benign and malignant variants. Am J Clin Pathol 2004; 122: 566-74.

2. Satyaprakash AK, Sheehan JS, Sangüez OP. Proliferating trichilemmal tumors: A review of the literature. Dermatol Surg 2007; 33: 1102-8.

\section{ATYPICAL MICROGLANDULAR ADENOSIS POST-NEOADJUVANT CHEMOTHERAPY}

Theresa Ly ${ }^{1}$, Renee C.F. Chan ${ }^{1}$, Norman Janu ${ }^{2}$, Michael Bilous

${ }^{1}$ Department of Anatomical Pathology, ${ }^{2}$ Breast and Endocrine Surgery, Concord Repatriation General Hospital, Concord, and

${ }^{3}$ Healthscope Pathology, NSW, Australia 